Präv Gesundheitsf 2011 · 6:83-84 DOI 10.1007/s11553-011-0293-2 Online publiziert: 19. März 2011 (c) Springer-Verlag 2011

\author{
U. Walter ${ }^{1} \cdot$ E. Pott ${ }^{2} \cdot$ T. Kliche ${ }^{3}$ \\ ${ }^{1}$ Institut für Epidemiologie, Sozialmedizin und Gesundheitssystemforschung, \\ Medizinische Hochschule Hannover, Hannover \\ ${ }^{2}$ Bundeszentrale für gesundheitliche Aufklärung, Köln \\ ${ }^{3}$ Institut und Poliklinik für Medizinische Psychologie, \\ Universitätsklinikum Hamburg-Eppendorf, Hamburg
}

\title{
Gesundheitsökonomie trifft Gesundheitsförderung und Prävention
}

\section{Ansätze und Weiterentwicklungen im BMBF- Förderschwerpunkt Präventionsforschung}

Mit der Charta von Tallinn erweiterte die WHO $2008 \mathrm{ihr}$ Gesundheitskonzept um eine ökonomische Perspektive. In der Präambel heißt es: „Neben ihrem Wert an sich trägt eine bessere Gesundheit durch ihre Auswirkungen auf wirtschaftliche Entwicklung, Wettbewerbsfähigkeit und Produktivität auch zum sozialen Wohlergehen bei ${ }^{\text {“1 }}$. Spätestens jetzt sind gesundheitsökonomische Betrachtungen in der Gesundheitsförderung und Prävention hoffähig.

Schon seit längerem werden in der gesundheitsbezogenen Versorgung nicht nur die Wirksamkeit von Maßnahmen, sondern auch die Kostenwirksamkeit als Nachweis gefordert. Damit müssen sich auch Prävention und Gesundheitsförderung Effizienzfragen stellen - und sie sind zunehmend in der Lage, dies zu tun. Eine wachsende Forschungsdichte für Teilfelder liefert belastbare Belege für die Wirksamkeit bestimmter Ansätze oder Maßnahmen und gestattet damit ihre gesundheitsökonomische Einschätzung.

Gesundheitsökonomische Methoden wurden vergleichsweise häufig auf (präventiv)medizinische Interventionen wie Impfungen und Screenings angewandt. Inzwischen liegen aber auch vermehrt Studien zur Kosteneffektivität für viele behaviorale Programme vor. Ebenso

${ }^{1} \mathrm{http}: / /$ www.euro.who.int/_data/assets/pdf_ file/0004/88609/E91438G.pdf. ist die gesundheitsökonomische Abschätzung von Interventionen in Settings, insbesondere in der betrieblichen Gesundheitsförderung, bei guter Forschungslage weiter vorangeschritten.

Immer wieder zeigen ökonomische Studien in der Prävention einen „return on investment" oder eine günstige Kosteneffektivität, wie - für Teilbereiche - etwa die jüngst veröffentliche OECD-Studie $\mathrm{zu}$ „obesity and the economics of prevention "2. Dennoch: die Gesamtlage ist heterogen und die Anforderungen an präventive und gesundheitsfördernde Interventionen sollten nicht höher angesetzt werden als für andere (namentlich therapeutische) Maßnahmen. So weisen Krauth, John und Suhrcke in ihrem Einführungsbeitrag darauf hin, dass Prävention nicht zu Kosteneinsparungen führen muss, aber die Kosteneffektivität vergleichbar mit etablierten medizinischen Technologien sein sollte.

Verbesserte gesundheitsökonomische Abschätzungen für Prävention und Gesundheitsförderung sind also dringend zu wünschen. Viele ökonomische Evaluationen unterbleiben bislang, weil die Versorgungsträger des Gesundheitswesens nicht unmittelbar selbst von ihnen profitieren. Dabei können gesundheitsökonomische Befunde eine bessere Orientie-

\footnotetext{
2 Franco Sassi (2010) Obesity and the economics of prevention. Fit not fat. OECD. www.oecd. org/publishing (31.01.2011).
}

rung für gesundheitspolitische Entscheidungen geben. Im Bereich der Prävention und Gesundheitsförderung scheint eine staatlich geförderte Forschung unabdingbar für die Förderung und Weiterentwicklung des Feldes zu sein.

Trotz der erfreulichen Entwicklungen bestehen zweifellos noch immer erhebliche methodische Anforderungen und Lücken für die Gesundheitsökonomie der Prävention. Sie betreffen besonders kontextabhängige, zieloffene und partizipative Vorgehensweisen, die schwer standardisierbar sind und deren Wirkungsbelege nicht durch Industrieforschung unterstützt werden. Zudem fehlen gesundheitsökonomische Studien zur Prävention aus gesamtgesellschaftlicher Perspektive.

Zur Schließung dieser Lücken leistet der Förderschwerpunkt Präventionsforschung des Bundesministeriums für Bildung und Forschung (BMBF) einen Beitrag. Dessen 6o Projekte arbeiten 20042012 an zentralen Querschnittsaufgaben: an neuen Vorgehensweisen und Zugangswegen, an Evidenzbasierung, Evaluation und Qualitätssicherung, an Forschungsplänen und Instrumenten für Prävention und Gesundheitsförderung und eben auch an gesundheitsökonomischen Methoden und Outcomes. Gesundheitsökonomische Evaluationen bilden ein Schlüsselthema für Leistungsfähigkeit und Transfer der Prävention. Das vorliegende Schwerpunktheft stellt dafür Erträge des 
BMBF-Förderschwerpunkts zusammen. Die (Projekt-)Beiträge berichten exemplarische Befunde, befassen sich mit der gesundheitsökonomischen Methodik und ihrer Weiterentwicklung und zeigen vordringliche forschungsstrategische Ansätze für eine bessere gesundheitsökonomische Evidenzlage zur Prävention auf.

- Einleitend geben Christian Krauth, Jürgen John und Marc Suhrcke einen Überblick über die Standards der gesundheitsökonomischen Evaluation und leiten Vorschläge für die Evaluation von Präventionsprogrammen im Vergleich zu medizinischen Interventionen ab. Die Autoren berücksichtigen dabei bestehende Besonderheiten wie langfristige Zeithorizonte, oft vielfältig eingebundene Akteure des Gesundheits-, Bildungs- und Sozialsystems und damit den sektorenübergreifend zu betrachtenden Nutzen und die Kosten der Interventionen.

- Im Vergleich zu klinischen, insbesondere pharmazeutischen Interventionen werden gesundheitsökonomische Evaluationen in der Prävention trotz einer Zunahme in den vergangenen zwei Jahrzehnten immer noch vergleichsweise selten durchgeführt. Ulla Walter, Martina Plaumann, Svenja Dubben, Guido Nöcker und Thomas Kliche geben, ausgehend von der Relevanz ökonomischer Betrachtungen, einen Einblick in die internationale und nationale Studienlage.

- Eine systematische Literaturrecherche von Anita Klein, Nadja Chernyak, Ralph Brinks, Jutta Genz und Andrea Icks zur Kosteneffektivität der Primärprävention von Diabetes mellitus Typ II ergab erfreulich viele Studien, die zeigen, dass die Prävention von Prädiabetes kosteneffektiv ist. Allerdings verdeutlicht die Analyse zugleich ein Dilemma: Trotz umgrenzter medikamentöser und verhaltensbezogener Interventionsansätze sind die Studien derart heterogen gestaltet, dass eindeutige Aussagen kaum getroffen werden können.

- Silke Röhl und Beate Schücking widmen sich der immer noch ungenügenden Jodversorgung bei Schwangeren und Stillenden in Deutschland. Über eine rechtzeitige Jodsubstitution ließen sich Krankheitskosten von gutartigen Schilddrüsenerkrankungen und ihrer Versorgung minimieren. Allerdings bestehen, wie eine von den Autorinnen durchgeführte Marktanalyse ergab, erhebliche Preisdifferenzen bei den Jodidpräparaten, auf die bei einer motivierenden Beratung hingewiesen werden sollte.

- Die gezielte Erreichung von vulnerablen Gruppen erfordert neue Wege und gilt als aufwändig. Christian Krauth, Okyta A'Walelu, Susanne Bisson, Ute Gerken, Wielant Machleidt, Ramazan Salman und Ulla Walter vergleichen die Kosteneffektivität von aktiven und passiven Zugangswegen zu russischsprachigen Migranten zur Suchtprävention. In der Studie wurde die übliche dichotome Einteilung von Zugangswegen differenziert, wobei sich Mischstrukturen als besonders kosteneffektiv erweisen.

- In der Betrieblichen Gesundheitsförderung werden zum einen Schätzungen von "return on investment" (ROI) unter Einbezug von Krankenstandskosten und Frühberentungen vorgenommen, zum anderen psychosoziale Effekte wie Arbeitszufriedenheit, Motivation und seelische Gesundheit mit ökonomischen Kennziffern des Betriebs in Beziehung gesetzt. Mit dem Ziel, die Relevanz dieser „weichen Faktoren“ für betriebliche Entscheidungen und den wirtschaftlichen Erfolg eines Unternehmens zu verdeutlichen, übersetzen Sigrun Fritz und Peter Richter den qualitativ erfassten Nutzen in finanzielle Parameter.

- ROI sind nur gültig für die jeweils berücksichtigten Rahmenbedingungen. Um eine Kosten-Nutzen-Einschätzung bei veränderten betrieblichen Kontexten vornehmen zu können, entwickelten Dieter Gloede und Antje Ducki ein praxisfähiges Kalkulationstool. Die Basis bildet ein in mehreren Betrieben erprobtes, modulares Gruppentraining zur Stressbewältigung für un- und angelernte Beschäftigte. Vor dem Hintergrund ihrer Analysen betrachten die Autoren ROI-Berechnungen als fragwür- dig und fordern eine methodische

Neuorientierung.

- Tina Salomon und Heinz Rothgang gehen der Frage nach, ob und wann gesundheitsökonomische Evaluationen zu einer Benachteiligung von älteren Menschen bei der Priorisierung von medizinischen Leistungen führen, und welche Auswirkungen sie auf den Bereich der Prävention und Gesundheitsförderung haben. Sie nehmen dabei u. a. die Perspektive der Analyse, qualitätsadjustierte Lebensjahre (QALY) sowie die indikationsübergreifende bzw. indikationsspezifische Anwendung von Methoden in den Blick.

Mit dem vorliegenden Schwerpunktheft möchten wir nicht nur neue Beiträge zu ökonomischen Analysen in der Prävention und Gesundheitsförderung bündeln, sondern zugleich einen Anstoß zu ihrer Weiterentwicklung und Diskussion liefern. Dieses Arbeitsfeld steht erst am Anfang. In den kommenden Jahren sind deutliche und produktive Entwicklungen zu erwarten, mit erheblichem Nutzen für Versorgungsträger, Gesundheitspolitik und Bevölkerung.

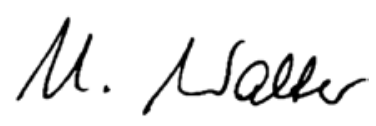

U. Walter

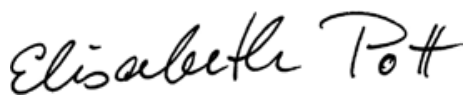

E. Pott

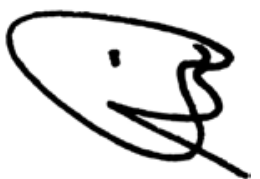

T. Kliche

\section{Korrespondenzadresse}

Prof. Dr. U. Walter

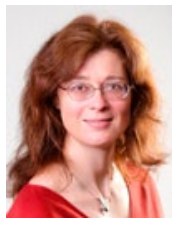

Institut für Epidemiologie, Sozialmedizin und Gesundheitssystemforschung, Medizinische

Hochschule Hannover

Carl-Neuberg-Straße 1, 30625 Hannover Walter.Ulla@MH-Hannover.de 